Relations industrielles

Industrial Relations

\title{
Le travail en équipes
}

Shift Work

\section{Pierrette Sartin}

Volume 22, numéro 3, 1967

URI : https://id.erudit.org/iderudit/027810ar

DOI : https://doi.org/10.7202/027810ar

Aller au sommaire du numéro

\section{Éditeur(s)}

Département des relations industrielles de l'Université Laval

\section{ISSN}

0034-379X (imprimé)

1703-8138 (numérique)

Découvrir la revue

Citer cet article

Sartin, P. (1967). Le travail en équipes. Relations industrielles / Industrial Relations, 22(3), 357-374. https://doi.org/10.7202/027810ar
Résumé de l'article

Après avoir indiqué les raisons d'ordre technique, économique et social du travail en équipes, l'auteur en expose les formes et l'organisation. Puis, il en souligne les principales difficultés familiales, sociales, et physiologiques. Enfin, il suggère des moyens pour améliorer la situation existante.
Tous droits réservés (C) Département des relations industrielles de l'Université Laval, 1967
Ce document est protégé par la loi sur le droit d'auteur. L'utilisation des services d'Érudit (y compris la reproduction) est assujettie à sa politique d'utilisation que vous pouvez consulter en ligne.

https://apropos.erudit.org/fr/usagers/politique-dutilisation/ 


\section{Le travail en équipes}

\section{Pierrette Sartin}

Après avoir indiqué les raisons dordre technique, économique et social du travail en équipes, lauteur en expose les formes et lorganisation. Puis; il en souligne les principales difficultés familiales, sociales, et physiologiques. Enfin, il suggère des moyens pour améliorer la situation existante.

Le travail en équipes encore appelé travail posté, qui pendant longtemps ne fut pratiqué que dans des travaux d'une nature particulière, comme les fonderies et les mines où il s'imposait, apparaît de plus en plus comme une nécessité économique et tend à se généraliser dans de nombreux secteurs industriels.

Les employés des grands magasins, les établissements de spectacles, de transports, les restaurants, les programmateurs de calculateurs électroniques et combien d'autres travailleurs y sont, eux aussi, soumis.

\section{Les raisons}

Un certain nombre de raisons d'ordre technique et économique, et même des raisons d'ordre social, font que ce travail est appelé à se développer de plus en plus, et il est nécessaire de mieux connaître ses avantages et ses dangers pour les prévenir et essayer de les pallier.

En effet la désynchronisation des rythmes de travail et des rythmes de la vie humaine s'accentue, ce qui ne va pas sans dommage pour l'homme et pour l'entreprise. Mais sur le volume de ce travail en équipes successives, on est encore peu éclairé et les statistiques ne reflètent que tardivement un mode de travail qui tend à s'étendre.

Les raisons d'ordre technique qui rendent nécessaire le travail posté sont bien connues et ce sont elles qui ont été à l'origine de son introduction. Certaines fabrications

SARTIN, Pierrette, Commissariat et Plan d'équipement et de productivité, Paris. 
en effet ne souffrent aucune interruption. C'est le cas de la sidérurgie, du travail du verre, des fours à chaux, de l'industrie chimique, du papier carton par exemple.

Dans beaucoup d'autres secteurs l'automation entraîne la continuité des processus de fabrication qui sont programmés et autocontrôlés.

De plus tout le travail d'entretien ne peut, le plus souvent se faire qu'en dehors des heures normales de travail des machines. Dans de nombreuses entreprises, même ne pratiquant pas le travail posté, il reste donc des secteurs qui, pour des raisons techniques, ne peuvent fonctionner que sur ce mode.

Mais ce sont les facteurs économiques qui sont les plus importants et qui déterminent l'augmentation actuelle du travail en équipes, car ces facteurs concernent toutes les branches de l'activité et non plus certains secteurs bien délimités.

D'une part, le matériel coûte de plus en plus cher; les investissements, et les amortissements, pèsent lourdement sur les coûts.

D'autre part, en raison des progrès techniques, ce matériel vieillit plus vite qu'il ne s'use: c'est le phénomène bien connu de l'obsolence. Plus une machine est perfectionnée, plus elle coûte cher; et plus vite aussi elle se périme.

C'est ainsi que le secteur des textiles qui connait de graves problèmes pour l'amortissement de son matériel, souvent dépassé et démodé avant même d'avoir été amorti et qui a, de ce fait, intérêt à le faire - tourner " pendant le plus grand nombre d'heures possible, voit se développer le travail en double et triple équipes.

L'\& amortissement s du matériel ne doit plus être calculé selon les règles fiscales, mais selon la réalité, c'est-à-dire en tenant compte de la rapidité du progrès technique. Le problème sera donc moins de maintenir ou d'augmenter la durée du travail de l'homme que de faire travailler la machine le plus longtemps possible... dans la mesure où l'on aura la possibilité d'écouler sa production. Et c'est en partie cette restriction qui limite le développement de lautomation.

Une enquête de la Direction des Prix faite en France il y a quelques années, montrait que dans beaucoup d'usines, le matériel conçu 
pour tourner 8,670 heures par an, tournait en réalité moins de 2,000 heures. Et l'on connaît dans de nombreuses entreprises, du matériel qui n'est pas utilisé au maximum de sa capacité.

Il y a donc là une véritable hérésie économique que la rationalisation des investissements peut sans doute diminuer mais non faire entièrement disparaître. Sur le plan de l'économie le problème majeur, avec celui du plein emploi de la main-d'oeuvre, est bien celui du plein emploi ou tout au moins de l'utilisation optimale des machines.

Ce problème de l'utilisation optimale des machines est particulièrement aigu dans les secteurs où l'on trouve à la fois un taux élevé de capitalisation, un matériel et une production en évolution constante. C'est le cas en particulier du textile où de gros efforts de modernisation ont dû être faits et où les méthodes de production sont en constant changement. C'est le cas aussi des travaux de bureau, avec l'introduction de l'électronique, et aussi de certains secteurs comme la chimie qui doivent faire face à des investissements coûteux et soumis à des renouvellements fréquents.

Enfin, un certain nombre de raisons d'ordre social incitent à travailler en équipes.

Il y a tout d'abord certains services publics qui ne souffrent pas d'interruptions ceux des chemins de fer, des postes, de la distribution d'eau et d'électricité, des hôpitaux, etc... Mais aussi, il y a la nécessité d'étaler les horaires, de pallier les pointes, d'augmenter la durée d'ouverture des magasins et de certains services, etc...

Pour toutes ces raisons, le travail en équipes répond à des besoins, voire à des nécessités qu'on ne saurait sous-estimer et il ne constitue plus comme par le passé un fait exceptionnel, mais bien * un mode de travail caractérisant un stade avancé de l'évolution technique, économique et sociale des sociétés industrielles ${ }^{\prime}{ }^{1}$

Examinons donc comment il apparait aujourd'hui, quelles formes il revêt, et après avoir vu ses avantages, quels inconvénients il recèle, au moins sous sa forme actuelle.

(1) Marc Maurice et Colette Montem. 


\section{Les. formes}

Une enquête psychosociologique faite par l'Institut des Sciences Sociales ${ }^{2}$, sous l'égide du Commissariat Général du Plan permet de dégager des enseignements dont la plupart dépassent le contexte français et qui à ce titre sont intéressants.

En France, le travail se présente encore comme assez rare puisque $25 \%$ seulement de l'ensemble des salariés travaillant dans les secteurs (industriel et commercial) couverts par l'enquête pratiquent ce mode de travail.

De plus, il apparait que ce travail existe surtout dans les grandes entreprises.

$3,4 \%$ des établissements de 10 à 20 salariés le pratiquent alors qu'il est de règle dans 59,4 des établissements occupant de 500 à 1000 salariés et de règle aussi dans $74,2 \%$ des établissements occupant de 1000 ouvriers et plus.

Mais si ce mode de travail revêt dans les grandes entreprises une certaine ampleur, il faut remarquer toutefois qu'il est très inégalement appliqué dans les différents secteurs industriels.

TABLEAU

LA DISTRIBUTION DES TRAVAILLEURS EN ÉQUTPE DANS LES DIFFÉRIENTS SECTEURS INDUSTRIELS

\section{Pourcentage des établissements pratiquant le travail en équipes}

Production des métaux

Industrie textile

Papier, carton

Industrie chimique, caoutchouc 26.1

Verre, céramique,

matériaux de construction $\quad 22.0$

\section{Pourcentage des salariés travaillant en équipes dans l'ensemble des établissements de chaque branche}

37.0

29.0

26.0

20.0

La proportion des ouvriers qui travaillent en équipes par rapport à l'ensemble des ouvriers occupés dans les seuls établissements qui pra-

(2) Par Marc Maurice et Colette Monterl. 
tiquent ce mode de travail est de $40,8 \%$ pour les industries de transformation.

Mais le pourcentage des salariés qui travaillent effectivement en équipes pour l'ensemble de ces mêmes industries ne dépasse pas $17 \%$, soit environ 1 salarié sur 6 .

Le travail en équipes ne concerne donc encore qu'une minorité de salaries; toutefois, il ne constitue plus une exception, limitée à quelques secteurs particuliers de l'industrie.

Il faut noter également que dans les pays, comme les Etats-Unis, l'Allemagne et les Pays-Bas où les industries sont plus concentrées qu'en France, ce mode de travail est plus largement développé.

\section{L'organisation}

En ce qui concerne l'organisation même de ce travail, diverses formules sont adoptées.

La journée « normale » s'étend sur 8 ou 9 heures selon que la semaine est répartie sur cinq jours, cinq jours et demie ou six jours.

Les horaires d'arrivée s'échelonnent entre $7 \mathrm{~h}$. et $8 \mathrm{~h}$. du matin: ceux de départ $17 \mathrm{~h}$. et $18 \mathrm{~h}$., selon le temps de la pause consacrée au déjeuner.

Le travail en deux équipes $(2 \times 8)$ suppose deux équipes alternant entre le poste du matin pendant un certain nombre de jours; et le poste de l'après-midi.

Ce système est assez homogène entre les différentes entreprises; seules les heures de prise et de fin de poste varient.

Les horaires les plus couramment pratiqués vont: de $6 \mathrm{~h}$. du matin à $14 \mathrm{~h}$. pour le poste du matin, et de $14 \mathrm{~h}$. à $22 \mathrm{~h}$. pour le poste de laprès-midi.

Dans le travail en trois équipes, les horaires varient selon que l'on travaille en équipes fixes, en semi-continu ou en continu, comme c'est généralement le cas dans le textile, c'est-à-dire en équipes faisant toujours le même poste. 
Les horaires courants sont alors: pour le poste du matin: de $5 \mathrm{~h}$. à $13 \mathrm{~h}$. pour le poste de l'après-midi: de $13 \mathrm{~h}$. à $21 \mathrm{~h}$. pour le poste de nuit: de $21 \mathrm{~h}$. a $5 \mathrm{~h}$. du matio.

Dans le textile, le problème des trois équipes soulève beaucoup de difficultés.

En effet, ce secteur emploie traditionnellement un fort pourcentage de main-d'oeuvre féminine qui vient souvent de régions minières éloignées et pour laquelle se posent la question du transport et celle de l'interdiction du travail de nuit qui, légalement, commence à $22 \mathrm{~h}$. La troisième équipe doit donc être composée de personnel masculin, et de nombreux établissements éprouvent aujourd'hui des difficultés à recruter de la main-d'oeuvre masculine pour cette troisième équipe, en raison notamment des salaires peu élevés des emplois sans qualification pratiqués dans une branche largement féminisée.

Dans le système en semi-continu, on trouve trois équipes et trois postes: un poste du matin, un poste de l'après-midi et un poste de nuit. La durée de chaque poste est généralement d'une semaine.

Les deux systèmes les plus couramment adoptés sont:

\section{Le semi-continu normal:}

pendant lequel chaque ouvrier tient alternativement chacun des postes (matin, après-midi et nuit) pendant une semaine. On passe donc du poste de nuit au poste du matin, avec un arrêt de 24 heures (du dimanche $4 \mathrm{~h}$. au lundi $4 \mathrm{~h}$.). Quand le passage se fait du matin a l'après-midi et de l'après-midi à la nuit, le repos est de 48 heures.

\section{Dans le semi-continu inversé:}

l'ordre de succession des trois postes est différent: on passe de l'après-midi au matin; du matin à la nuit; de la nuit à l'après-midi.

On a ainsi, soit un arrêt de 32 heures; soit un arrêt de 56 heures (dans le cas d'arrêt entre le poste du matin et celui de la nuit).

Le nombre d'heures de repos en fin de cycle est dans les deux cas le même; mais leur répartition diffère et peut être ressentie différemment sur le plan psychologique. 
On rencontre généralement ces horaires dans la sidérurgie, dans la construction automobile et dans certains secteurs de la chimie.

Le système des trois équipes en continu qui se rencontre aussi dans la construction automobile et dans la chimie est en réalité pratiqué avec quatre équipes: trois équipes travaillant dans les postes du matin, de l'après-midi et de la nuit pendant que la quatrième équipe se repose.

Ce système est le plus complexe et un grand nombre de combinaisons peuvent être adoptées en jouant sur la fréquence des changements de postes, le sens de leur rotation, la durée du cycle, le nombre d'heures de repos consécutives et la répartition de ces heures de repos dans le cycle; enfin sur le nombre de dimanche et de jours fériés libérés dans le cycle.

On peut avoir ainsi:

- un régime de 7 demi-équipes, comme dans la sidérurgie et les acieries Thomas, les Hauts fournaux, l'entretien,

- un régime $4 \times 8$ normal, se décomposant en 42 jours de travail et 14 jours de repos,

- un régime de $4 \times 8$ inversé,

- un régime de $4 \times 8$ doublé,

- un régime de $4 \times 7$ normal ou doublé, etc...

\section{Les difficultós}

La complexité de ces systèmes tient à la fois aux problèmes et aux habitudes propres à chaque industrie, mais aussi aux difficultés que rencontre leur application et notamment aux préférences des salariés pour certains horaires.

Ces difficultés sont a la fois d'ordre familial, social, physiologique.

Sur le plan familial, le travail en équipe engendre ou peut engendrer, une désynchronisation des activités et des rythmes de vie, notamment en ce qui concerne les heures de repas, de sommeil, de liberté des enfants. Il engendre aussi des difficultés dans l'exercice des responsabilités familiales notamment en matière d'éducation et ces difficultés sont fortement ressenties par la majorité des travailleurs. 
Les effets nocifs de cette désynchronisation s'accentuent lorsque le travail comporte un poste le dimanche ou certains jours de fête comme Noël ou Pâques qui, non seulement représentent des fêtes familiales, mais aussi des jours de congé attendus et désirés par la majorité des salariés.

Les compensations financières atténuent les "gênes ? et les frustrations éprouvées, sans toutefois les effacer.

Sur le plan social, c'est la difficulté d'entretenir avec les amis une vie de relation qui est le plus fortement ressentie. Le travailleur en équipes se sent en effet très souvent en "marge "; "socialement mort ? comme on l'a souvent écrit; en marge de la vie de société, de la vie du syndicat et aussi de la vie de l'entreprise. Il est souvent tenu à l'écart des informations et dans les entreprises où fonctionnent à la fois le travail en équipe et le travail normal, il se produit entre les groupes une sorte de clivage.

Enfin, il est difficile au travailleur en équipe de participer a la vie syndicale, à la vie des associations ou des groupements.

Mais la gêne la plus importante qu'il perçoit est celle qu'il rencontre dans l'utilisation de ses loisirs. Les loisirs en effet sont organisés en fonction de la journée normale traditionnelle et le travailleur en équipes se trouve coupé à la fois des activités de formation faites par l'entreprise et qui lui offriraient une chance de promotion et des activités capables de le distraire et de le détendre.

Dans l'enquête effectuée par l'Institut des Sciences Sociales du travail, la hiérarchie des gênes ressenties en allant de la plus contraignante à la moins contraignante était:

1 - La gêne dans la vie familiale,

2 - La gêne dans la vie de loisirs,

3 - La gêne dans les relations avec les amis,

4 - La gêne dans la vie à l'usine,

5 - La gêne dans la participation à des associations,

6 - La gêne dans les déplacements, les courses. 
En dépit de ces gênes, d'une manière générale, et d'après les enquêtes faites, le travail en double équipe est assez bien accepté, sauf toutefois:

- par les femmes qui n'aiment pas les déplacements dans l'obscurite;

- par les pères de famille qui ne peuvent veiller à l'éducation de leurs enfants;

- par les travailleurs âgés de plus de quarante-cinq ans qui trouvent les 8 heures continues trop fatigantes;

- par ceux qui éprouvent le besoin d'avoir une vie sociale que ces horaires rendent souvent impossible.

Mais en plus des inconvénients signalés qui, par le gêne, les soucis et parfois le mécontentement qu'ils engendrent, sont une cause de fatigue supplémentaire, il faut citer les cas beaucoup plus graves, d'horaires incompatibles avec le temps normal de sommeil:

- De 5 heures à 13 heures, par exemple, et de 13 heures à 21 heures, ou fréquemment aussi de 4 heures à 13 heures et de 13 heures aे 22 heures.

A ces horaires s'ajoutent la longueur du trajet jusqu'au lieu de travail, les difficultés du a ramassage organisé tant bien que mal par les entreprises, à des heures où ne fonctionne aucun service de transports en commun, dans les localités pas ou mal desservies par ceux-ci, ou encore les mauvaises conditions physiques dans lesquelles se trouve celui qui va prendre son poste à une heure anormale, et les risques d'accidents qui en résultent quand il utilise des moyens de transport individuels.

En ce qui concerne les inconvénients sur le plan de la santé, les dangers du travail en équipe ont donné lieu à de nombreuses controverses.

Il faut distinguer toutefois le travail en deux ou trois équipes avec travail de nuit, et surtout le travail en équipes fixes ou en équipes alternantes. 
Tout d'abord, il faut noter, qu'à quelques exceptions près, il a été vérifié par les physiologistes que la disposition au travail était plus favorable au poste du matin. Elle est moins bonne au poste de l'aprèsmidi et tout à fait défavorable au poste de nuit, vers les 3 heures du matin.

De plus le travail de nuit contrevient aux lois normales de la physiologie, notamment en ce qui concerne l'inversion de la température, plus basse normalement la nuit que le jour, quand l'individu se repose et cède au sommeil. C'est le renversement de la température centrale qui crée, pour l'organisme, la principale difficulté à s'adapter à un rythme nouveau.

Les recherches faites sur la circulation par Menzel ${ }^{3}$ montrent qu'il y a une déficience générale de la circulation dans les heures nocturnes.

A 3 heures du matin, l'organisme est dans un état impropre au travail corporel ou intellectuel. Cela est si vrai que le rendernent des équipes de nuit est en moyenne inférieur de 2 a $17 \%$ à celui des équipes de jour.

Or, en dépit d'une adaptation superficielle aux circonstances, ceux qui ont effectué un travail de nuit pendant des années ont néanmoins ce rythme minimal et cette déficience de 3 heures du matin.

De plus, le travail de nuit nécessite un effort d'adaptation particulier, effort que certains individus ne parviennent jamais à faire. Pour que le repos diurne pût être aussi complet et réparateur que le repos nocturne, il nécessiterait en outre des conditions de silence et de logement qui, à l'heure actuelle, sont rarement réalisées.

Une enquête de sept ans faite au Danemark, en Norvège et en Suède, tous pays où les conditions de logement sont, dans l'ensemble, supérieures à celles de la France et assez comparables à celles existant au Québec, est particulièrement significative à cet égard.

Le tableau ci-dessous indique le pourcentage de cas d'insomnie chez les sujets habitant leur propre maison et chez ceux qui sont logés dans de mauvaises conditions.

(3) Menzel, Sur la physiologie et la pathologie du travail de nuit et d'équipes s. Arbeisphysiologie, 14, 504, 1950. 
TABLEAU \|

LES TRAVAMLEURS TOUChÉs PAR L'INSOMNIE

\section{$\%$ d'insomnie}

Habitant leur Mauvaises conditions maison d'habitation

Travailleurs à la journée 12 18

Travailleurs en équipe 15 73

Travailleurs à la journée qui, auparavant, travaillaient en équipe

Dans la journée normale de travail, la durée moyenne du sommeil est de 7 à 8 heures.

En équipe de $2 \times 8$ au poste du matin: $41 \%$ des sujets dorment 6 à 7 heures, et $34 \%$ dorment de 7 à 8 heures.

Dans les équipes en $3 \times 8$ et $4 \times 8,24 \%$ des sujets dorment 5 heures et moins.

Pour le poste du matin, $40 \%$ des individus en $3 \times 8$ et $33 \%$ des individus en $4 \times 8$ dorment de 6 a 7 heures.

Pour les postes de l'après-midi, $41 \%$ des individus en $3 \times 8$ et $36 \%$ en $4 \times 8$ dorment de 7 a 8 heures.

Néanmoins, même dans des conditions imparfaites, et exception faite des individus qui ne parviennent absolument pas à inverser leur rythme, le travail de nuit, s'il est constant, peut être effectué sans dommage. L'expérience montre que les accidents du travail en particulier ne sont pas plus nombreux chez les ouvriers travaillant constamment de nuit que chez les autres. Pas plus nombreux non plus chez ceux travaillant en équipe que chez ceux qui travaillent à la journée.

Le travail de nuit, en outre, est souvent très bien accepté par les jeunes, satisfaits d'avoir des loisirs dans l'après-midi et dans la soirée, au détriment souvent de leur santé; mieux accepté encore, au point de créer des rivalités, quand il s'accompagne d'une prime supplémentaire et d'une surveillance plus relâchée.

Le travail de nuit, dans la quasi totalité des pays est interdit sauf dérogation aux femmes et celles-ci commencent à réclamer que cette interdiction soit levée. 
C'est assurément un vaste problème et qui ne peut être abordé ici... Disons seulement que les nuisances du travail de nuit sont les mêmes pour les hommes et pour les femmes et que, sous réserve de celles qui ne le supportent pas, ses conséquences ne sont, en soi, pas plus fâcheuses pour elles que pour eux.

Cependant deux éléments doivent être pris en considération en ce qui concerne les femmes:

- d'une part, le fait que la main-d'oeuvre féminine constituant une main-d'oeuvre mal défendue, on peut, par des moyens détournés, lui imposer en fait, ce mode de travail. En dépit de la sévérité de la réglementation, cela se fait trop souvent en France sans que les pouvoirs publics puissent intervenir, toutes les infractions à la législation du travail se faisant grâce à la complicité de l'employeur et du travailleur.

- d'autre part la charge domestique qui repose principalement sur la femme risque de se trouver aggravée par le travail de nuit. On peut craindre que la femme qui «sent» devant elle son travail domestique a faire, ou ses devoirs maternels à remplir, parvienne encore moins que lhomme, à trouver le repos nécessaire... Et comme elle est, nerveusement plus résistante que l'homme, il faut s'attendre à ce que, sauf des moyens exceptionnels de prévention, (psychologiques autant que législatifs) elle dépasse la limite de ses forces.

Même bien accepté, le travail de nuit ou seulement en double équipe n'en présente pas moins des dangers sérieux pour la santé des travailleurs.

Les plus fréquents des troubles ressentis sont les troubles gastriques.

La fréquence des maux d'estomac dans l'enquête faite par l'Institut des Sciences Sociales est au maximum de $18 \%$ en journée normale, de $37 \%$ en équipe $2 \times 8$, de $35 \%$ en équipe $3 \times 8$, de $44 \%$ en équipe $4 \times 8$.

La cause majeure du déséquilibre physique et nerveux qui s'insinue lentement dans l'organisme réside moins dans la double et la triple équipe que dans les horaires tournants, c'est-à-dire dans les changements hebdomadaires ou bi-hebdomadaires d'équipes, qui en sont, généralement, la conséquence. 
Dans le rapport qu'elle a présenté aux Journées d'Etudes de la Ligue française d'Hygiène mentale, la Commission d'études des rythmes de travail a précisé qu'on pouvait évaluer à $70 \%$ la proportion des ouvriers supportant mal ou très mal l'horaire tournant.

Une enquête faite dans une usine de 800 ouvriers montre que l'on compte trois fois plus d'ulcères gastriques dans le personnel faisant les $3 \times 8$ que parmi les ouvriers faisant la journée normale.

En ce qui concerne l'irritabilité et la nervosité, l'enquête de l'Institut des Sciences Sociales montre que si l'irritabilité est un trouble important puisqu'elle atteint près des $3 / 4$ de la population enquêtée, les différences selon les horaires pratiqués sont minimes. Mais le type d'horaire le plus atteint est celui des équipes en $4 \times 8$ où $71 \%$ de la population souffre de nervosisme.

Une enquête faite à Bordeaux dans les centres industriels de la gironde, sous la direction du professeur Derville, a montré que le système $3 \times 8$ était généralement mal supporté, notamment par les femmes et les sujets jeunes.

\section{Les horaires tournants}

Le docteur Paul Sivadon, directeur à l'office mondial de la santé, estime que les horaires tournants sont non seulement à l'origine de nombreuses névroses et maladies organiques, mais encore de l'alcoolisme. Les professions les plus touchées par l'alcoolisme seraient aussi celles qui ont des horaires courants avec travail de nuit, surtout si le rythme est trop court pour permettre toute adaptation.

Sur la nocivité des horaires tournants, à quelques divergences de détail près, tous les médecins, physiologistes et psychiâtres de travail, tant en France qu'à l'étranger, sont d'accord.

Dans la meilleure hypothèse, cette adaptation ne peut se faire qu'au bout d'un temps assez long. Dans le cas des ouvriers français, il y a impossibilité absolue d'une adaptation puisque ceux-ci sont obligés à une alternance plus ou moins rapide (hebdomadaire ou bi-hebdomadaire, ou, au mieux, tous les quinze jours). En tout cas, trop rapidement pour que l'organisme puisse s'adapter au changement d'horaire et reprendre un rythme nouveau. 
De plus, ce changement hebdomadaire d'équipe qui empêche, l'organisme de trouver le rythme de vie régulier qui lui est nécessaire, s'accompagne souvent de a doublage " en fin de cycle, ce qui donne une fois par semaine, dans beaucoup d'entreprises, une journée continue de 16 heures. Il y a donc là une source très grande de fatigue et un danger que l'on a tort de sous-estimer. Par ailleurs, les médecins du travail ont pu observer qu'il n'y avait pas de système tournant satisfaisant et qu'une désadaptation dans le sommeil se produisait nécessairement... On a remarqué en effet que les salariés changeant d'équipe au bout d'un mois présentaient des troubles du sommeil dès la 3ème semaine; de même qu'ils en présentaient dès la 2ème semaine quand le changement avait lieu par période de 21 jours etc... II semble bien que ce point n'ait pas été entièrement élucidé sur le plan physiologique et sans doute y aurait-il là toute une recherche à faire afin de connaître les incidences psychologiques qui viennent perturber l'adaptation.

C'est pourquoi et dans l'état actuel de nos connaissances il est difficile de trouver des remèdes aux dangers que font courir aux travailleurs les horaires tournants. Mal informés des risques qu'encourt leur santé ou indifférents à ceux-ci, les ouvriers les préfèrent et surtout ceux qui saccompagnent de changements hebdomadaires. Certains horaires sont plus recherchés que d'autres, soit à cause de la liberté qu'ils laissent dans la journée et qui est employée à des travaux de jardinage, bricolage, les uns et les autres ajoutant une fatigue supplémentaire à celle du travail, soit à cause des primes et autres avantages qui compensent le travail de nuit.

Dans l'ensemble, le poste préféré est, pour des raisons diverses, celui du matin. Mais il faut remarquer que dans les enquêtes faites, les individus manifestaient surtout un attachement relativement fort au système quils pratiquaient et auquel ils étaient habitués.

Les horaires tournants apparaissent souvent comme plus justes à la majorité des salariés.

De leur côté, les chefs d'entreprise qui sentent la nécessité d'utiliser au mieux leur outillage, d'accroître leur production et qui ne sont pas davantage informés de la nocivité de ces horaires tournants sont peu enclins à courir les risques d'un conflit pour imposer d'autres horaires ou trouver d'autres formules.

Dès lors, que peut-on faire? Allonger jusquà trois semaines les périodes d'alternance des horaires pour permettre à l'organisme de 
s'adapter, comme cela se fait en Angleterre? Mais c'est un palliatif bien incertain. Jusqu'à trois mois comme cela se pratique souvent en Amérique? Raccourcir, au contraire, les cycles et admettre qu'une nuit sur deux, ou deux sur quatre, le sujet rompt le rythme normal de sa vie, comme cela se pratique en France dans certains services publics? Mais c'est créer un état de déséquilibre constant, une fatigue qui cherche son remède dans l'alcool, et qui se traduit, en dernière analyse, par des accidents et des troubles divers, aussi dommageables pour l'individu que pour la collectivité.

\section{Conclusion}

Une solution pourrait être trouvée, en utilisant quatre équipes au lieu de trois, l'équipe de nuit ne faisant plus que 4 heures au lieu de 8; on pourrait d'ailleurs faire appel pour celle-ci, à défaut d'autre maind'oeuvre, à des réadaptés qui ne peuvent supporter 8 heures de travail, ou à des retraités de l'industrie qui accepteraient un travail de nuit de brève durée pour compléter leur pension de retraite, souvent insuffisante. En Angleterre où le même problème s'est posé, on utilise des non-professionnels dans toutes les entreprises ou professions où cela est possible et on essaie pour eux de généraliser le travail à mi-temps.

On pourrait d'ores et déjà améliorer la situation existante:

- En évitant de faire travailler en équipe les ouvriers qui demeurent loin de leur travail ou qui vivent dans des conditions de logement ou de famille difficilement compatibles avec celui-ci.

- En renforçant la surveillance médicale de ces travailleurs, notamment de ceux qui font du travail de nuit.

- En ne mettant pas en équipe ceux qui ne peuvent médicalement s'y adapter ou les travailleurs âgés habitués à travailler de jour et avec des horaires fixes.

- Enfin en faisant un effort d'information médicale très pousée pour mettre employeurs et salariés au courant des dangers que présentent les horaires tournants.

Il parait certain que l'on s'achemine vers une extension de plus en plus grande de cette forme de travail. Aussi faut-il essayer de l'adapter aux possibilités de l'homme, car l'inverse ne sera jamais possible. Les impératifs de l'économie ne parviendront pas à modifier les lois de la physiologie et c'est pourquoi la recherche doit être poursuivie pour déterminer les véritables nuisances du travail posté et une action psy- 
chologique et d'information être entreprise pour amener dans toute la mesure du possible la suppression des horaires tournants.

\section{SHIFT WORK}

INTRODUCTION

Shift work more and more appears to be an economical necessity and seems to spread into many industrial sectors - almost everybody is submitted to it. However, it is necessary to have a better knowledge of the advantages and dangers of that kind of work in order to prevent and to palliate them.

\section{Its Causes}

The technical reasons that are the origin of that kind of work are well known. But economical factors are much more important and determine the actual growth of shift work - Those factors are :

a) the cost of equipment is higher;

b) because of progress, equipment grows older more rapidly than its real depreciation.

However there are a fow social reasons for shift work :

a) a few public services cannot be stopped;

b) the necessity of lenghtening opening hours of stores, etc.

Shift work is thus a characteristic of an advanced period of the technical, economical and social evolution of industrial societies.

\section{ITs FonMs}

Eventhough that kind of work is quite used in large firms, note is to be taken that shift work has different forms depending of the industrial sector.

The General Commisariate of the French Plan has published in its survey a graphic that clearly shows the mentioned differences.

TABLE I

The Distribution of Shift-workers in the Industrual Sectors

\section{Percentage of firms using} shift work

Metal production

60.4

Textile industry

Paper industry

Chemical industry

Building material
$\%$ of wage earners working on shifts in every sector
55.0

37.0

29.0

26.0 
As a general rule, let us say that in France, one wage-earner out of six works on shifts. Thus only a minority of wage-earners are implied in that kind of work; however it is not an exception anymore limited to some industrial sectors.

\section{Its Organtzation}

That are mainly two sorts of shift work : the two team and the three team shift-work :

1) In the two team shift work, the system is quite homogeneous : only beginning and ending hours of work varry. The most fluently practiced time table is the following :

6 a.m. - 2 p.m. - morning team;

2 p.m. - 10 p.m. - afternoon team.

2) In the three team shift work, in the textile industry, for example, the usual time tables are :

5 a.m. - 1 p.m. - morning team;

1 p.m. - 9 p.m. - afternoon team;

9 p.m. - 5 a.m. - night team.

However, in the textile industry, the percentage of women employed brings out many difficulties.

Three systems of organization exist :

1.-In the first system, the worker changes of shift each. week.

2.-In the second, the order of succession of the shifts is different : from P.M. to A.M., from A.M. to night shift and from night shift to P.M.

3.- The third system officially requires three teams to cover the twenty four hours. In fact, this system needs four teams : three teams are working while the fourth is at rest.

The complexity of three problems is in fact due to the difficulties and practices of each industry and is also due to the difficulties met in their application and to the preference of wage-earners to certain time tables.

Those difficulties are at the same time :

a) familial

- change in normal life customs.

- difficulties in practicing familial responsabilities.

b) social

- difficulties in keeping in relations with friends.

- the shift-worker feels \& social dead $₫$.

- he feels on the fringe of the union, the firm and society.

- he cannot take advantage of formation and leisure program.

c) physiological.

The dangers of shift work are quite discussed. Note is to be taken that it has 
been proven that workers are more inclined to work on the morning shift than on any other shift. In fact, as Menzel puts it, there is a general deficiency of blood circulation during night time.

On the other hand, daily rest, in order to be as complete and refreshing as night rest, would necessitate silence and lodging facilities that are quite rare at the present time especially in France.

The following table on insomnia illustrates my idea :

TABLE II

Worxers AFFected by INSOMNA

\% affected by insomnia

$\begin{array}{lcc} & \begin{array}{c}\text { Lioing at their } \\ \text { place }\end{array} & \begin{array}{c}\text { Bad lodging } \\ \text { conditions }\end{array} \\ \text { Day-labourer } & 12 & 18 \\ \text { Shift workers } & 15 & 75 \\ \text { Day-labourer having been shift-worker } & 84 & 97\end{array}$

However, two points must be taken into consideration about women in the labor force :

a) shift work is often imposed to them and, because of their non-unionization, it is difficult for them to resist;

b) night shift affects domestic duties of women.

Note is to be taken that the major cause of physiological and nervous desiquilibrium is the frequent changes in time tables. Thus keeping the body from finding a regular life rythm so necessary.

\section{CONCLUSTON}

One could solve the problem by using four teams instead of three : the night shift would not work more than four hours. To do so, it would be preferable to employ half time workers especially for those night shifts.

The present situation could be ameliorated:

a) by avoiding to ask workers living far from the firm or having bad lodging conditions to work on night shifts ;

b) by increasing medical supervision of these workers;

c) by avoiding to place on work shift those who cannot medically get adapted to it or older workers used to work daily on fixed time tables;

d) by making a special effort to give medical information to employers and wage earners on the dangers of changing time-tables.

It seems that we are inclined to a greater use of this kind of work. We must try to adapt it to man. The contrary is impossible. Even economics will never modify physological laws. That is why research must be continued in order to determine the dangers of shift work. In addition to this, an information campaign must be undertaken in order to eliminate changing time-tables. 